\title{
siRNA-Mediated RNA Interference in Precision-Cut Tissue Slices Prepared from Mouse Lung and Kidney
}

\author{
Mitchel J. R. Ruigrok, ${ }^{1}$ Nalinie Maggan, ${ }^{1}$ Delphine Willaert, ${ }^{1}$ Henderik W. Frijlink, ${ }^{1}$ Barbro N. Melgert, ${ }^{2}$ \\ Peter Olinga, ${ }^{1,3}$ and Wouter L. J. Hinrichs ${ }^{1}$
}

Received 12 July 2017; accepted 17 August 2017; published online 11 September 2017

\begin{abstract}
Small interfering RNA (siRNA)-mediated RNAi interference (RNAi) is a powerful post-transcriptional gene silencing mechanism which can be used to study the function of genes in vitro (cell cultures) and in vivo (animal models). However, there is a translational gap between these models. Hence, there is a need for novel experimental models that combine the advantages of in vitro and in vivo models (e.g., simplicity, flexibility, throughput, and representability) to study the effects of siRNA. This need may be addressed by precision-cut tissue slices (PCTS), which represent an ex vivo model that mimics the structural and functional characteristics of a whole organ. The goal of this study was to investigate whether self-deliverable siRNA (Accell siRNA) can be used in precision-cut lung slices (PCLuS) and precision-cut kidney slices (PCKS) to achieve RNAi ex vivo. PCLuS and PCKS were prepared from mouse tissue, and they were subsequently incubated up to $48 \mathrm{~h}$ with no siRNA (untransfected), non-targeting Accell siRNA, or Gapdhtargeting Accell siRNA. Significant Gapdh mRNA silencing was achieved (PCLuS 55\%; PCKS $\sim 40 \%$ ) without compromising the viability and morphology of slices. Fluorescence microscopy confirmed that Accell siRNA diffused into PCLuS and PCKS. Spontaneous inflammation upon incubation was observed in PCLuS and PCKS as shown by a higher mRNA expression of proinflammatory cytokines $I l 1 b, I l 6$, and Tnfa, although Accell siRNA appeared to diminish this response in PCLuS after $24 \mathrm{~h}$. In conclusion, this ex vivo transfection model can be used to evaluate the effects of siRNA in relevant biological environments.
\end{abstract}

KEY WORDS: ex vivo; gene silencing; siRNA; tissue slices; transfection.

\section{INTRODUCTION}

Small interfering RNA (siRNA)-mediated RNA interference (RNAi) leads to the degradation of specific

\footnotetext{
${ }^{1}$ Groningen Research Institute of Pharmacy, Department of Pharmaceutical Technology and Biopharmacy, University of Groningen, Antonius Deusinglaan 1, 9713 AV, Groningen, The Netherlands.

${ }^{2}$ Groningen Research Institute of Pharmacy, Department of Pharmacokinetics, Toxicology, and Targeting, University of Groningen, Antonius Deusinglaan 1, 9713 AV, Groningen, The Netherlands.

${ }^{3}$ To whom correspondence should be addressed. (e-mail: p.olinga@rug.nl)

Abbreviations: 18 s $18 \mathrm{~S}$ ribosomal RNA, ANOVA Analysis of variance, ATP Adenosine triphosphate, Birc5 Baculoviral inhibitor of apoptosis repeat-containing 5, Gapdh Glyceraldehyde-3-phosphate dehydrogenase, H\&E Hematoxylin and eosin, Illb Interleukin 1 beta, Il6 Interleukin 6, KHB Krebs-Henseleit buffer, PBS Phosphate-buffered saline, PCKS Precision-cut kidney slices, PCLuS Precision-cut lung slices, PCR Polymerase chain reaction, PCTS Precision-cut tissue slices, PKR Protein kinase R, PRR Pattern recognition receptor, RIG-1 Retinoic-inducible gene 1, RNAi RNA interference, TLR Toll-like receptor, Tnfa Tumor necrosis factor alpha, UW University of Wisconsin, mRNA Messenger RNA, qPCR Real-time quantitative polymerase chain reaction, siRNA Small interfering RNA
} of a specific protein [1]. To that end, siRNA can be used, for instance, to decrease the expression of disease-related genes, to investigate the function of proteins, or to establish experimental models of disease. Therefore, the use of siRNA to induce RNAi in vitro (cell cultures) and in vivo (animal models) has been described in various publications [2-4]. However, there is a big translational gap between in vitro and in vivo models. In vitro models are known for their simplicity, flexibility, and high throughput but they lack a biological context. Conversely, in vivo models generate more biologically relevant data although they are more complex to use. Hence, there is an obvious need for novel experimental models that combine the advantages of in vitro and in vivo models to study the effects of siRNA.

This need may be addressed by using precision-cut tissue slices (PCTS). PCTS refer to viable explants, with a welldefined thickness and diameter, prepared from tissue (e.g., lung and kidney) that can be cultured ex vivo [5, 6]. In comparison to conventional in vitro models, PCTS offer crucial advantages. Firstly, PCTS comprise the structural and functional heterogeneity of tissues, including cell-matrix interactions and intercellular interactions. Moreover, 
interspecies comparisons regarding biological effects of siRNA are possible as PCTS can be prepared from animal and human tissue. PCTS can also be quickly prepared in a simple and reproducible manner with specialized equipment that maintains the viability of tissue during the slicing process. In addition, several experiments can be performed per animal as multiple PCTS can be prepared from a single piece of tissue. Lastly, PCTS can be incubated for up to 48-120 h, depending on the tissue type and species, which means they can be used to study endogenous metabolism, drug effects, and disease mechanisms [5, 7-9]. As such, PCTS appear to have potential as a novel experimental model to study the effects of siRNA.

Nevertheless, RNAi in PCTS requires the successful delivery of siRNA into the cytoplasm of cells. Because siRNA is negatively charged and does not readily pass cell membranes, researchers often use cationic nanocomplexes (e.g., polyplexes, lipoplexes, or lipopolyplexes) to achieve RNAi in vitro and in vivo [10]. Unfortunately, such nanocomplexes have a limited penetration into PCTS which appears to be dependent on particle size and electrostatic interactions between the particle and surrounding proteins; this could lead to aggregation of nanocomplexes [11,12]. To overcome this obstacle, self-deliverable siRNA could be used. Selfdeliverable siRNA, which refers to siRNA conjugated with cell-penetrating peptides or sterols, requires no complexation with polymers or lipids to enable transport into cells [13]. The extent of diffusion through biological tissues is thought to be greater for self-deliverable siRNA as it is smaller than conventional nanocomplexes. Some publications have described the successful induction of RNAi in vivo upon administration of self-deliverable siRNA [14, 15]. Accordingly, PCTS could be an ideal model to timely assess effects of self-deliverable siRNA in both animal and human tissue.

Thus, the principal aim of this study was to investigate whether self-deliverable siRNA (Accell siRNA) can successfully silence mRNA expression in precision-cut lung slices (PCLuS) and precision-cut kidney slices (PCKS) prepared from mouse tissue. These organs were selected as PCLuS and PCKS represent non-solid and solid tissue. First, fluorescence microscopy and real-time quantitative polymerase chain reaction (qPCR) was used to assess the diffusion of Accell siRNA into slices and to determine whether RNAi was successfully induced, respectively. Next, we analyzed the adenosine triphosphate (ATP) content and morphology of slices to determine whether slices remained viable during transfections. Lastly, we studied the mRNA expression profile of pro-inflammatory cytokines (i.e., Illb, Il6, and Tnfa) to determine whether spontaneous inflammation develops upon incubation and whether Accell siRNA can affect this (foreign nucleic acids can induce activation of the innate immune system) [16].

\section{MATERIALS AND METHODS}

\section{Animal Tissue}

Tissue was obtained from C57BL/6J mice, which were housed under controlled conditions with a 12-hour light/dark cycle and free access to water and food (Central Animal
Facility, University Medical Center Groningen, Groningen, The Netherlands). The lungs and kidneys were extracted in a terminal procedure conducted under isoflurane $/ \mathrm{O}_{2}$ anesthesia (Nicholas Piramal, London, UK). Note, prior to being extracted, the lungs were inflated in situ with liquefied and pre-warmed $\left(37^{\circ} \mathrm{C}\right)$ support medium containing $1.5 \%$ lowgelling-temperature agarose type VII (Sigma-Aldrich, Zwijndrecht, The Netherlands) and $0.9 \% \mathrm{NaCl}$ (Merck, Darmstadt, Germany) to provide cutting support during the slicing process [5]. After extraction, organs were immediately placed in ice-cold University of Wisconsin (UW) preservation solution (DuPont Critical Care, Waukegab, USA). All experiments were approved by the Animal Ethical Committee of the University of Groningen (permit no. 6416AA).

\section{Preparation of Slices}

After the organ extraction, slices were prepared with a Krumdieck tissue slicer (Alabama Research and Development, Munford, USA), which was filled with ice-cold KrebsHenseleit buffer (KHB) supplemented with $25 \mathrm{mM}$ D-glucose (Merck), $25 \mathrm{mM} \mathrm{NaHCO}$ (Merck), and $10 \mathrm{mM} \mathrm{HEPES} \mathrm{(MP}$ Biomedicals, Aurora, USA); saturated with carbogen (95\% $\mathrm{O}_{2}$ and $5 \% \mathrm{CO}_{2}$ ); and adjusted to a $\mathrm{pH}$ of 7.4 [5]. To prevent loss of viability, slices were immediately transferred to icecold UW preservation solution after slicing. PCLuS and PCKS had a wet weight of 5-6 and 4-5 mg, respectively, with an estimated thickness of $250-350 \mu \mathrm{m}$ and a diameter of $\sim 5 \mathrm{~mm}$.

\section{Incubation of Slices}

Slices were incubated individually in a $1-\mathrm{mL}$ pre-warmed and oxygenated culture medium at $37^{\circ} \mathrm{C}$ under $5 \% \mathrm{CO}_{2}$ and $80 \% \mathrm{O}_{2}$, while gently shaken (90 cycles per minute). PCLuS culture medium was composed of Accell siRNA Delivery Media (GE Dharmacon, Lafayette, USA) supplemented with $100 \mathrm{U} / \mathrm{mL}$ penicillin-streptomycin (Life Technologies, Bleiswijk, The Netherlands), $50 \mu \mathrm{g} / \mathrm{mL}$ gentamicin (Life Technologies), and $0.1 \mathrm{mM}$ non-essential amino acids (Life Technologies). PCKS culture medium was composed of Accell siRNA Delivery Media (GE Dharmacon) supplemented with $10 \mu \mathrm{g} / \mathrm{mL}$ ciprofloxacin (Fresenius Kabi, Zeist, The Netherlands), and $10 \mathrm{mM}$ glucose (Sigma-Aldrich).

\section{Transfection of Slices}

After the slicing procedure, slices were either directly sampled or pre-incubated for $2 \mathrm{~h}$ in culture medium, as described in the previous section. Slices were subsequently transferred to the 1-mL pre-warmed and oxygenated culture medium supplemented with $10 \mu \mathrm{L}$ Accell siRNA buffer (GE Dharmacon), fluorescent red non-targeting Accell siRNA (GE Dharmacon), non-targeting Accell siRNA pool (GE Dharmacon), or Gapdh-targeting Accell siRNA pool (mouse) (GE Dharmacon). Accell siRNA buffer contained $60 \mathrm{mM}$ $\mathrm{KCl}, 6 \mathrm{mM}$ HEPES (pH 7.5), and $0.2 \mathrm{mM} \mathrm{MgCl}_{2}$. Gapdhtargeting Accell siRNA was used to study specific mRNA silencing while non-targeting Accell siRNA was used to identify potential off-target effects. PCLuS culture medium contained 0 or $0.5 \mu \mathrm{M}$ siRNA, whereas PCKS culture 
medium contained 0 or $1.0 \mu \mathrm{M}$ siRNA. After 24 or $48 \mathrm{~h}$ of incubation, slices were collected for analysis.

\section{Fluorescence}

Confocal laser scanning microscopy was used to assess the diffusion of red fluorescent non-targeting Accell siRNA into slices. Upon sampling, slices were briefly washed $(\sim 5 \mathrm{~s})$ in ice-cold phosphate-buffered saline (PBS), embedded in KPCryoCompound (Klinipath, Duiven, The Netherlands), and stored at $-80^{\circ} \mathrm{C}$ until use. Cross sections of $4 \mu \mathrm{m}$ were prepared from the slices by using a cryostat at $-20^{\circ} \mathrm{C}$. The sections were afterwards dried for $30 \mathrm{~min}$ at room temperature in the dark. Thereafter, ProLong Diamond Antifade Mountant with DAPI (Fisher Scientific, Landsmeer, The Netherlands) was applied to the sections, which were subsequently covered with glass coverslips. The sections were then dried for $24 \mathrm{~h}$ at room temperature in the dark. Sections were stored at room temperature in the dark until further use. Images were taken with a Leica TCS SP8 confocal microscope (Leica Microsystems, Rijswijk, The Netherlands). Imaging of DAPI and red fluorescent nontargeting Accell siRNA (DY-547) was done through excitation using laser lines at wavelengths of 405 and $552 \mathrm{~nm}$, respectively.

\section{mRNA Expression}

Silencing and inflammatory effects of Accell siRNA on mRNA expression were determined by qPCR. After transfection, three slices of the same condition were pooled, snap-frozen in liquid nitrogen, and stored at $-80^{\circ} \mathrm{C}$ until further use. As columnbased RNA isolation procedures are incompatible with agarosecontaining slices, total RNA of PCLuS was extracted using the Maxwell 16 LEV SimplyRNA Tissue Kit (Promega, Leiden, The Netherlands), whereas total RNA of PCKS was isolated with the FavorPrep Tissue Total RNA Mini Kit (Bio-Connect, Huissen, The Netherlands) [17]. Isolated RNA (750 ng) was reverse transcribed with the Reverse Transcription System (Promega), using an Eppendorf mastercycler $\left(25^{\circ} \mathrm{C}\right.$ for $10 \mathrm{~min}$, $45^{\circ} \mathrm{C}$ for $60 \mathrm{~min}$, and $95^{\circ} \mathrm{C}$ for $5 \mathrm{~min}$ ). Next, the mRNA expression was analyzed by qPCR, using specific primers (Table I) and FastStart Universal SYBR Green Master (Roche, Almere, The Netherlands). The qPCR analysis was carried out with a ViiA7 real-time qPCR (Applied Biosystems, Bleiswijk, The Netherlands), using 1 cycle of $10 \mathrm{~min}$ at $95^{\circ} \mathrm{C}$ and 40 cycles of $15 \mathrm{~s}$ at $95^{\circ} \mathrm{C}, 30 \mathrm{~s}$ at $60 \mathrm{C}$, and $30 \mathrm{~s}$ at $72 \mathrm{C}$. Finally, mRNA expression was calculated as fold induction with the $2^{-\Delta \Delta C t}$ method, using $18 \mathrm{~s}$ as reference gene.

\section{ATP Content}

The ATP content in slices was quantified to determine whether Accell siRNA affected the viability. After transfection, slices were placed individually in tubes containing $1 \mathrm{~mL}$ of sonication solution (70\% ethanol and 2 mM EDTA). The samples were subsequently snap-frozen in liquid nitrogen and stored at $-80^{\circ} \mathrm{C}$ until use. Next, the ATP content was analyzed with the ATP Bioluminescence Kit (Roche Diagnostics, Mannheim, Germany), as described previously [5]. Obtained ATP values (pmol) were normalized with respect to the total amount of protein $(\mu \mathrm{g})$ which was determined with the RC DC Protein Assay (Bio Rad).

\section{Morphology}

Morphological analyses were performed to assess whether Accell siRNA affected the structural integrity of the slices. After the transfection, slices were fixed in $4 \%$ formalin at $4^{\circ} \mathrm{C}$ for $24 \mathrm{~h}$. Next, slices were dehydrated in ethanol baths with increasing concentrations. Slices were subsequently cleared in xylene baths. Thereafter, slices were horizontally embedded in paraffin and sectioned $(4 \mu \mathrm{m})$. Prior to staining with hematoxylin and eosin (H\&E), sections were deparaffinized and rehydrated. The microscopic appearance of slices was assessed by evaluating the cytoplasm and the shape/staining of nuclei. Sections were scanned with a C9600 NanoZoomer (Hamamatsu Photonics, Hamamatsu City, Japan).

\section{Statistics}

Statistics were performed with GraphPad Prism 6.0 using one-way analyses of variance (ANOVA) followed by post hoc Tukey's multiple comparisons tests. Differences between groups were considered to be statistically significant when $p<.05$. Note, qPCR data was analyzed statistically by using the $\Delta \mathrm{Ct}$ values, although the data is shown as fold induction $\left(2^{-\Delta \Delta C t}\right)$.

\section{RESULTS}

\section{siRNA Penetration into PCTS}

Confocal laser scanning microscopy was used to confirm whether Accell siRNA penetrates into PCLuS and PCKS after $48 \mathrm{~h}$ of incubation (Fig. 1). Nuclei were stained with DAPI (blue) and showed a staining pattern consistent with morphology data (i.e., apoptotic regions at proximal tubules in PCKS). More importantly, fluorescent non-targeting Accell siRNA (red) was able to diffuse into PCLuS and PCKS, though the extent of diffusion was greater in PCLuS than in PCKS.

\section{siRNA-Mediated RNAi in PCTS}

qPCR analyses were performed to determine whether siRNA-mediated RNAi occurred in PCLuS and PCKS after being incubated with no siRNA (untransfected), nontargeting Accell siRNA, or Gapdh-targeting Accell siRNA

Table I. Primer Details

\begin{tabular}{llc}
\hline Gene & Forward sequence $\left(5^{\prime}-3^{\prime}\right)$ & Reverse sequence $\left(5^{\prime}-3^{\prime}\right)$ \\
\hline $18 s$ & CTTAGAGGGACAAG & ACGCTGAGCCAGTC \\
& TGGCG & AGTGTA \\
Gapdh & ACAGTCCATGCCAT & GATCCACGACGGAC \\
& CACTGC & ACATTG \\
Illb & TGAGCACCTTCTTT & TTGTCTAATGGGAA \\
& TCCTTCA & CGTCACAC \\
Il6 & TGATGCTGGTGACA & TAAGCCTCCGACTT \\
& ACCACGGC & GTGAAGTGGTA \\
Tnfa & CTGTAGCCCACGTC & TTGAGATCCATGCC \\
& GTAGC & GTTG
\end{tabular}



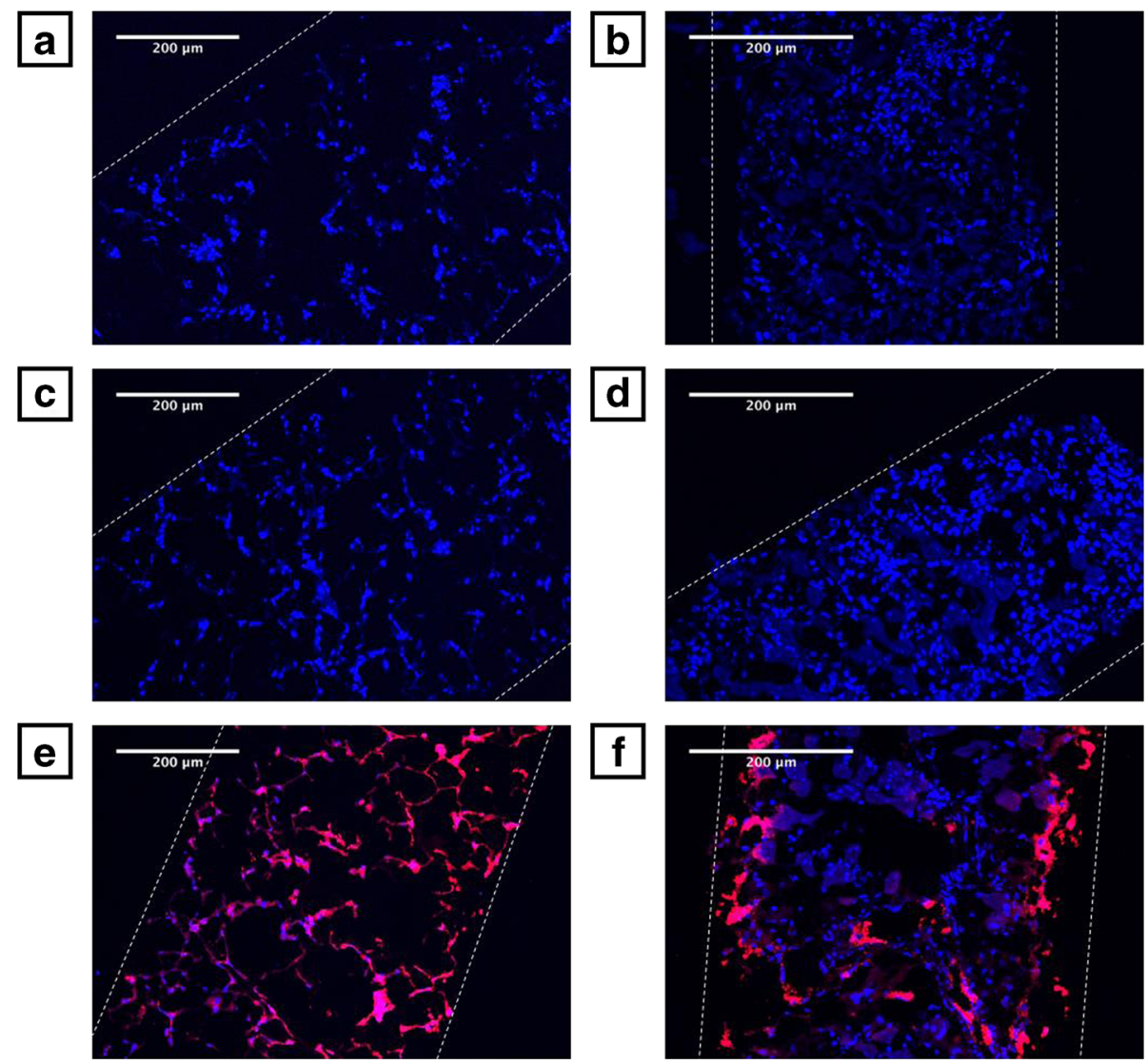

Fig. 1. siRNA penetration into PCTS. Slices were untransfected (a PCLuS, b PCKS) or treated with non-targeting Accell siRNA (c PCLuS, d PCKS) or fluorescent red non-targeting Accell siRNA (e PCLuS, f PCKS) for $48 \mathrm{~h}(n=4)$. Slices were subsequently collected for analysis by confocal laser scanning microscopy. Cross sections $(4 \mu \mathrm{m})$ were prepared with a cryostat and mounted with ProLong Diamond Antifade Mountant with DAPI (blue). Representative images

(magnification 20×) are shown of independent experiments performed in triplicate

for $48 \mathrm{~h}$ (Fig. 2). In PCLuS and PCKS, Gapdh-targeting Accell siRNA significantly lowered Gapdh mRNA expression by $\sim 55$ and $\sim 40 \%$, respectively, compared to untransfected slices. Furthermore, non-targeting Accell siRNA did not significantly change Gapdh mRNA expression in PCLuS and PCKS.

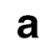$$
\text { a }
$$

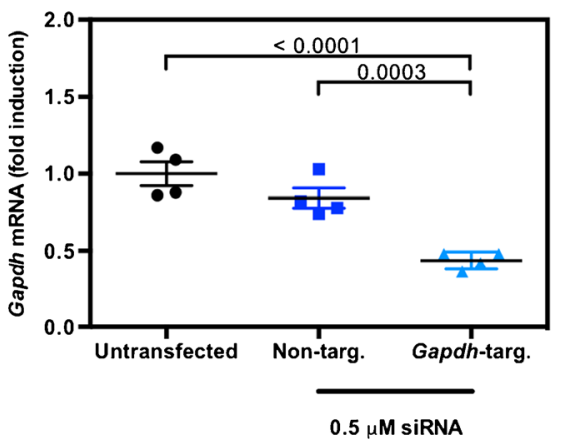

\section{ATP Content During siRNA Transfections}

The ATP content in PCLuS and PCKS was quantified to check whether Accell siRNA could negatively affect the viability after culturing slices up to $48 \mathrm{~h}$ (Fig. 3). No significant changes were observed in ATP content between

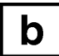

b

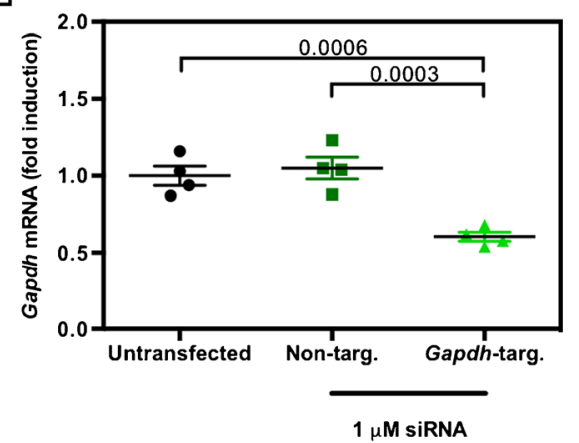

Fig. 2. siRNA-mediated RNAi in PCTS. a PCLuS and b PCKS were untransfected or treated with nontargeting Accell siRNA or Gapdh-targeting Accell siRNA for $48 \mathrm{~h}(n=4)$. Thereafter, Gapdh mRNA expression was analyzed by qPCR. $18 s$ was used as reference gene. Values represent individual experiments performed in triplicate and are accompanied with the arithmetic mean (horizontal line) \pm standard error of the mean (error bars). Differences were statistically significant when $p<.05$ (one-way ANOVA, post hoc Tukey's multiple comparisons test) 
a

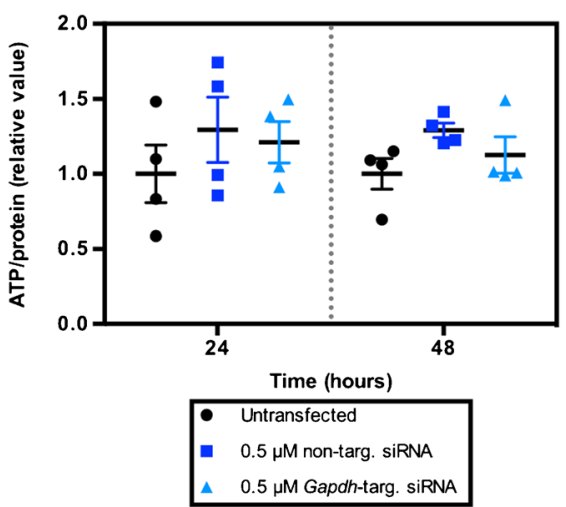

b

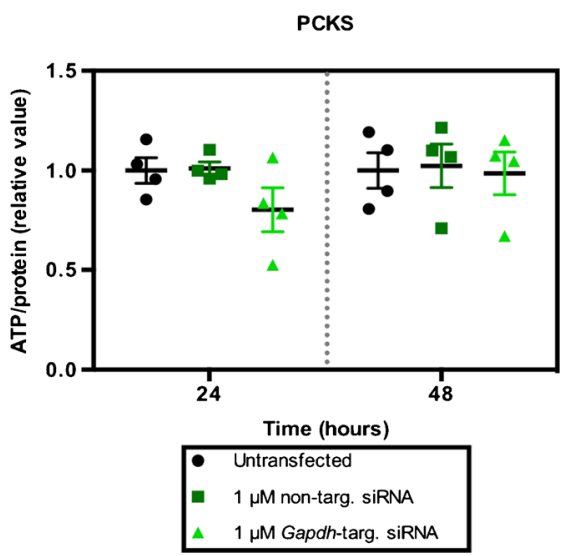

Fig. 3. ATP content during siRNA transfections. a PCLuS and b PCKS were untransfected or treated with non-targeting Accell siRNA or Gapdh-targeting Accell siRNA for 24 and $48 \mathrm{~h}(n=4)$, after which the ATP content was determined. Values (relative to untransfected slices, which are set to 1.0, at 24 and $48 \mathrm{~h}$ ) represent individual experiments performed in triplicate and are accompanied with the arithmetic mean (horizontal line) \pm standard error of the mean (error bars). No significant differences were observed (oneway ANOVA, post hoc Tukey's multiple comparisons test)

PCLuS and PCKS treated with no siRNA, non-targeting Accell siRNA, and Gapdh-targeting Accell siRNA.

\section{Morphology After siRNA Transfections}

The morphology of PCLuS and PCKS was evaluated to identify whether Accell siRNA affected the structural integrity of slices after $48 \mathrm{~h}$ of incubation (Fig. 4). As shown, PCLuS maintained their structural integrity. No substantial differences were observed between untransfected slices and slices treated with non-targeting Accell siRNA or Gapdhtargeting Accell siRNA. In general, PCKS maintained their structural integrity after $48 \mathrm{~h}$ of incubation as well, with no differences between the treatment groups, though minor signs of cellular damage (e.g., cellular swelling and nuclear dissolution) were observed in proximal tubules.

\section{Spontaneous Inflammation in Untransfected PCTS}

qPCR analyses were also performed to determine whether spontaneous inflammation occurred in untransfected PCLuS and PCKS (Fig. 5). To that end, mRNA expression of $I l 1 b$, Il6, and Tnfa was studied over time (0, 24, and $48 \mathrm{~h})$. In PCLuS, mRNA expression of these pro-inflammatory cytokines was found to significantly increase over time. Tnfa mRNA expression in PCKS had a similar pattern as in PCLuS. However, in PCKS, different patterns were observed in $I l 1 b$ and $I l 6$ mRNA expression as it significantly increased during the first $24 \mathrm{~h}$ of culturing, after which it appeared to decrease again.

\section{Pro-inflammatory Cytokines in Transfected PCTS}

The impact of Accell siRNA on the mRNA expression of pro-inflammatory cytokines in PCLuS and PCKS was studied by qPCR as well (Fig. 6). After $24 \mathrm{~h}$, non-targeting Accell siRNA and Gapdh-targeting Accell siRNA significantly decreased the mRNA expression of Illb and Tnfa in PCLuS by approximately $50 \%$, as compared to untransfected slices. Additionally, in comparison to untransfected PCLuS, PCLuS treated with non-targeting Accell siRNA had a significantly lower $(\sim 50 \%)$ Il6 mRNA expression after $48 \mathrm{~h}$. In contrast, non-targeting Accell siRNA and Gapdh-targeting Accell siRNA did not significantly affect mRNA expression of proinflammatory cytokines in PCKS after an incubation up to $48 \mathrm{~h}$.

\section{DISCUSSION}

In this study, we set out to explore whether RNAi could be achieved in PCLuS and PCKS from mouse tissue. It was found that both PCLuS and PCKS were successfully transfected with Gapdh-targeting Accell siRNA. This resulted in significant silencing of Gapdh mRNA expression, without affecting the viability, and with limited effects on inflammation.

\section{Slice Transfection}

To induce RNAi in PCTS, diffusion of Accell siRNA into slices is required. In this study, it was demonstrated that fluorescent Accell siRNA diffused into PCLuS and PCKS, though the extent of diffusion was greater in PCLuS. Thereafter, we showed that RNAi was successfully induced in PCLuS and PCKS with Accell siRNA as significantly lower Gapdh mRNA expression was observed in slices treated with Gapdh-targeting Accell siRNA. Non-targeting Accell siRNA did not induce significant changes in Gapdh mRNA expression, suggesting no off-target effects. Preliminary experiments showed lower siRNA concentrations did not result in Gapdh mRNA silencing, whereas higher concentrations induced offtarget effects (data not shown). A previous publication on polyplex-mediated siRNA transfections in PCTS prepared from tumor xenografts (solid tissue) described similar mRNA silencing ( $50 \%$ after $96 \mathrm{~h}$ of incubation with $1 \mu \mathrm{M}$ siRNA) but then for Birc5 mRNA [12]. However, their transfection technique cannot be compared to the technique presented here (siRNA polyplexes vs. self-deliverable siRNA) as different target mRNAs (Birc5 vs. Gapdh) and different tissue types (tumor tissue vs. primary tissue) were used. We 

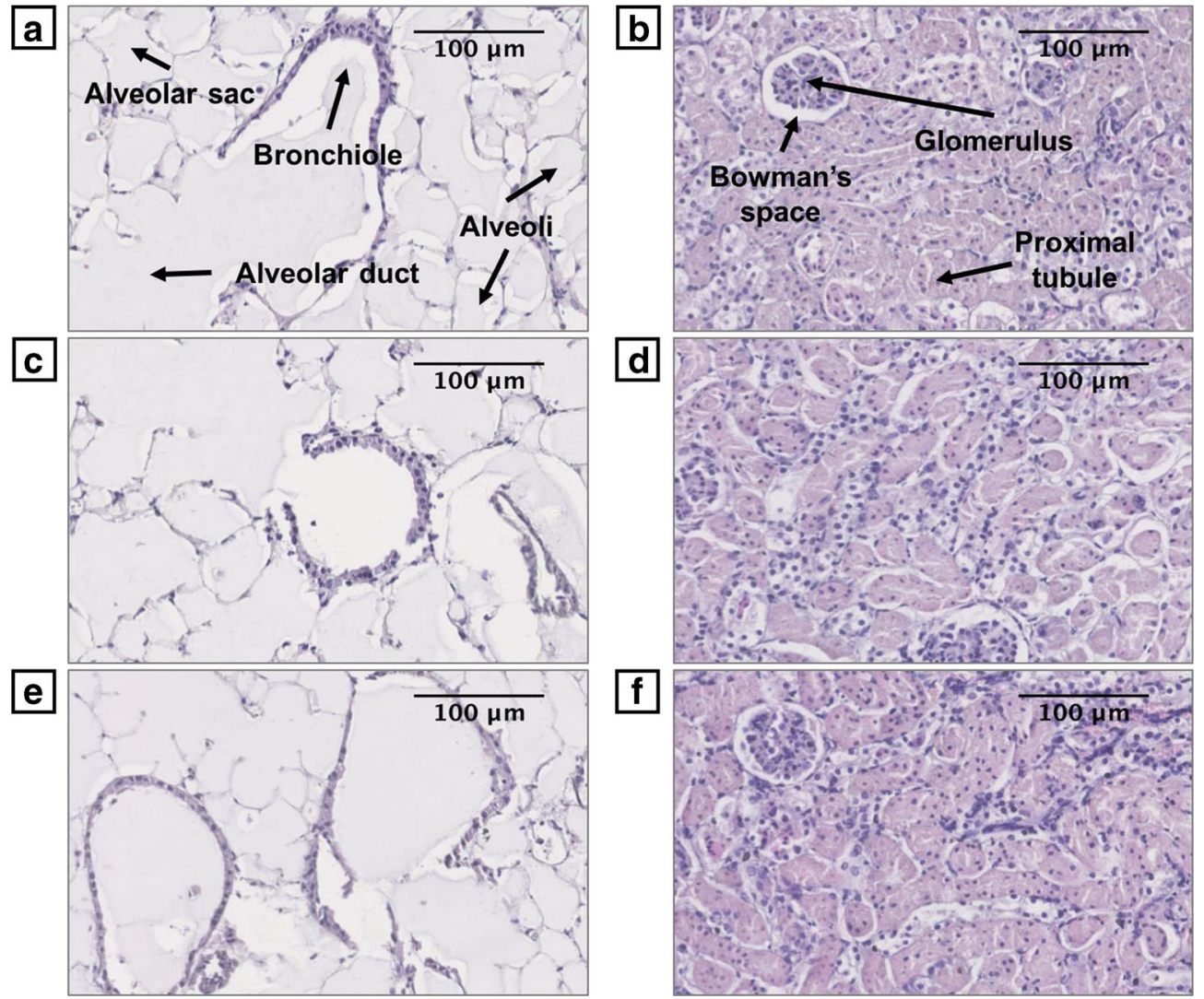

Fig. 4. Morphology after siRNA transfections. Slices were untransfected (a PCLuS, b PCKS) or treated with non-targeting Accell siRNA (c PCLuS, d PCKS) or Gapdh-targeting Accell siRNA (e PCLuS, f PCKS) for $48 \mathrm{~h}(n=4)$. Thereafter, slices were collected for morphological analysis. Paraffin sections $(4 \mu \mathrm{m})$ were stained with H\&E and subsequently scanned. Representative images (magnification 20×) are shown of independent experiments performed in triplicate

also attempted to transfect PCTS with Lipofectamine RNAiMAX, which is a commonly used lipid transfection reagent to transfect continuous cell lines, but we did not observe any mRNA silencing (data not shown). However, such reagents have a limited transfection efficiency in primary cells [18]. As PCTS contain primary cells, it may have been the case that lipoplexes were unable to be taken up via endocytosis or to diffuse into PCTS. Accell siRNA does not depend on endocytosis and, consequently, can lead to RNAi in PCTS. In this present study, however, it appears a transfection was achieved in the majority of cells that are present in the slices, though further research is necessary to obtain direct evidence. To that end, immunohistochemistry could be used to identify if the transfection with siRNA was uniform. In addition, by using a double-staining for cellspecific markers, immunohistochemistry could be used to show which cells are affected by the siRNA [19]. As GAPDH is an intracellular house-keeping enzyme, further research on specific cell-matrix and cell-cell interactions was beyond the scope of this study, though such interactions could be assessed in future studies with, for instance, in situ polymerase chain reaction (PCR) [20].

Interestingly, differences were observed in Gapdh mRNA silencing and siRNA penetration between PCLuS and PCKS. PCKS required a higher concentration of Accell siRNA than PCLuS to produce mRNA silencing effects (1 vs. $0.5 \mu \mathrm{M})$. This might be caused by differences in the number of cells and the number of cells per unit volume. PCKS are prepared from solid tissue, whereas PCLuS are prepared from non-solid lung tissue inflated with agarose, which accounts for a large volume in slices. As a result, PCKS contain more cells than PCLuS [5]. Furthermore, the greater number of cells per unit volume in PCKS might have also affected the penetration of Accell siRNA by restraining the diffusion of siRNA from a region of high concentration to a region of low concentration. Researchers have previously shown that fluorescent lysozyme $(\sim 15 \mathrm{kDa})$ diffused into PCKS prepared from mice [21,22]. As such, it is not surprising that Accell siRNA, which has a comparable molecular weight to the fluorescent lysozyme, could also diffuse into PCKS. Unlike PCKS, PCLuS contained an agarose matrix with a pore size that enabled rapid diffusion of Accell siRNA, thereby resulting in a higher mRNA silencing. Though there are differences in the penetration of Accell siRNA between PCLuS and PCKS, it remains striking that 55\% Gapdh mRNA silencing in PCLuS requires $0.5 \mu \mathrm{M}$ Accell siRNA, whereas only $40 \%$ Gapdh mRNA silencing in PCKS requires $1.0 \mu \mathrm{M}$ Accell siRNA. This suggests that the outermost cells in PCKS were more extensively transfected than the outermost cells in PCLuS. Nonetheless, the precise cellular and intracellular localization of Accell siRNA remains largely unclear and should be addressed in future research.

However, in neither PCLuS nor PCKS was GAPDH protein expression affected by Gapdh-targeting Accell siRNA 

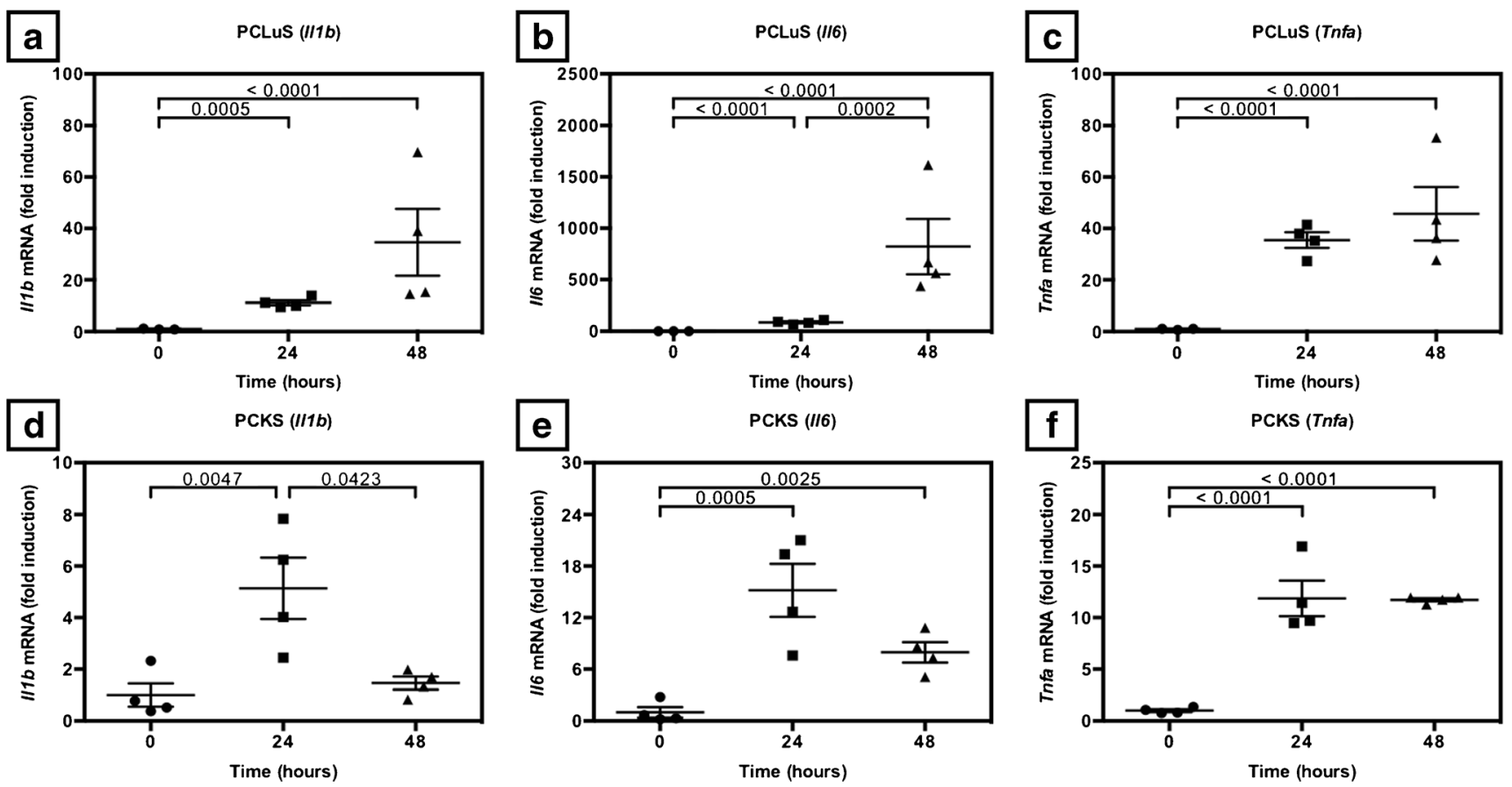

Fig. 5. Spontaneous inflammation in untransfected PCTS. Untransfected PCLuS and PCKS were sampled directly after slicing $(0 \mathrm{~h})$ and after an incubation of 24 or $48 \mathrm{~h}(n=3-4)$. Thereafter, mRNA expression of Il1b (a PCLuS, d PCKS), Il6 (b PCLuS, e PCKS), and Tnfa (c PCLuS, f PCKS) was analyzed by qPCR. $18 \mathrm{~s}$ was used as reference gene. Values represent individual experiments performed in triplicate and are accompanied with the arithmetic mean (horizontal line) \pm standard error of the mean (error bars). Differences were statistically significant when $p<.05$ (one-way ANOVA, post hoc Tukey's multiple comparisons test)

after $48 \mathrm{~h}$ of incubation, as determined by Western blotting expression and GAPDH protein expression could have arisen (data not shown). The discrepancy between Gapdh mRNA from differences in their intracellular half-lives. The half-life
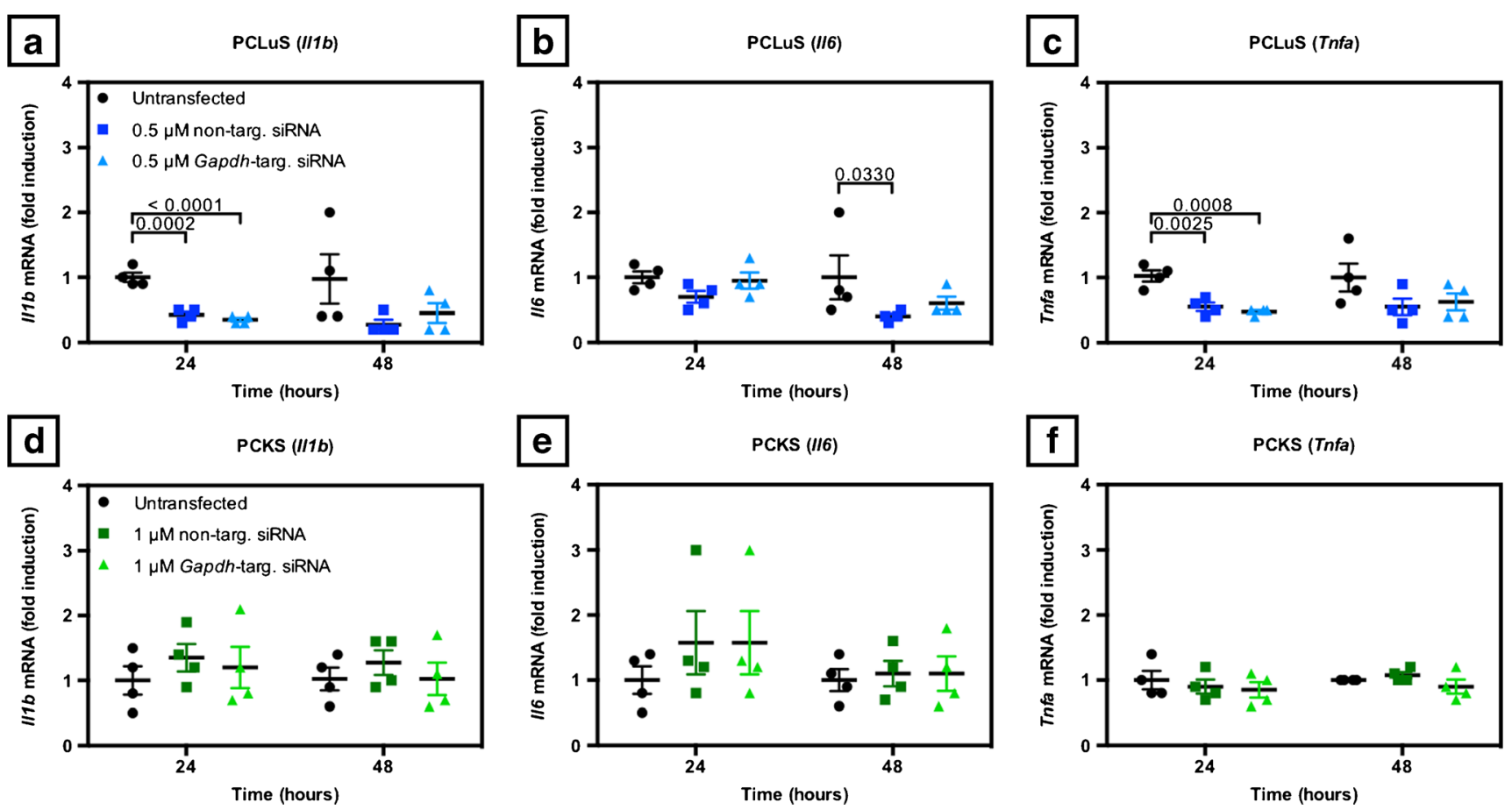

Fig. 6. Pro-inflammatory cytokines in transfected PCTS. PCLuS and PCKS were untransfected or treated with non-targeting Accell siRNA or Gapdh-targeting Accell siRNA for 24 and $48 \mathrm{~h}(n=4)$. Afterwards, the mRNA expression of $I l 1 b$ (a PCLuS, d PCKS), Il6 (b PCLuS, e PCKS), and Tnfa (c PCLuS, f PCKS) was analyzed by qPCR. $18 s$ was used as reference gene. Values (relative to untransfected slices, which are set to 1.0 , at 24 and $48 \mathrm{~h}$ ) represent individual experiments performed in triplicate and are accompanied with the arithmetic mean (horizontal line) \pm standard error of the mean (error bars). Differences were statistically significant when $p<.05$ (one-way ANOVA, post hoc Tukey's multiple comparisons test) 
of Gapdh mRNA in HeLa cells has been previously demonstrated to be $\sim 8 \mathrm{~h}$, whereas the half-lives of GAPDH protein in NRK-52E and A549 cells have been shown to be greater than $35 \mathrm{~h}$ [23-25]. This could mean that equilibration between Gapdh mRNA and protein levels requires more time because reductions in mRNA do not directly affect protein concentrations. Thus, it is worthwhile to select proteins with a quick turn-over or to extend the incubation time to allow for equilibration between mRNA and protein. However, extending the incubation time would require the optimization of incubation conditions in order to prevent myofibroblast overgrowth in PCLuS and to prevent apoptosis of the proximal tubules in PCKS.

\section{Slice Viability}

The effects of Accell siRNA on the viability of PCLuS and PCKS was studied from a biochemical and morphological perspective. In both PCLuS and PCKS, no significant differences in ATP content were observed between untransfected slices and slices treated with non-targeting Accell siRNA and Gapdhtargeting Accell siRNA. This indicates that the viability of slices was not affected. These results are in line with the morphology of the slices. PCLuS maintained all morphological features after $48 \mathrm{~h}$ of incubation. However, both untransfected and transfected PCKS contained some apoptotic proximal tubules (marked by loss of nuclei and swelling of cells), although the general morphology remained unaffected. Apoptosis in proximal tubules has been reported previously, and the authors suggested it is caused by a disturbed pre-urine flow [8,22]. As such, observed morphological changes in PCKS are not attributable to the siRNA.

\section{Slice Inflammation}

We also showed that spontaneous inflammation developed in untransfected PCLuS and PCKS, as marked by a significant increase in mRNA expression of pro-inflammatory markers $I l l b$, Il6, and Tnfa during incubation [16]. Conversely, in PCKS, mRNA expression of $I l 1 b$ and $I l 6$ appeared to decrease after $24 \mathrm{~h}$, suggesting partial resolution. Nonetheless, cytokine production in PCTS is usually not as pronounced as immune responses in vivo because immune cells cannot be recruited from the blood [26]. As such, the extent of immune responses in PCTS is dependent on the number and type of immune cells in the tissue slice. The rootcause of this inflammatory response in slices is not fully understood, though it probably results from mechanical (e.g., slicing procedure itself) and chemical injury (e.g., warm/cold ischemia) which can lead to a wound-repair response [27]. Previous literature reported similar responses in precision-cut intestinal slices (PCIS) prepared from rat, mouse, and human tissue [28]. Furthermore, PCKS prepared from human tissue have also been characterized by inflammation upon incubation for up to $48 \mathrm{~h}$ [8].

We also studied whether Accell siRNA had an effect on the spontaneous inflammation in PCLuS and PCKS. In general, unmodified siRNA can activate the innate immune system via siRNA sequence-dependent or sequenceindependent recognition by pattern recognition receptors (PRR) such as toll-like receptors (TLR) 3, 7, and 8; protein kinase R (PKR); and retinoic-inducible gene 1 (RIG-1) [16].
Activation of these receptors sets off an immune signaling cascade which leads to an inflammatory response characterized by an increased mRNA expression of pro-inflammatory cytokines $I l 1 b$, Il6, and Tnfa. Interestingly, non-targeting and Gapdh-targeting Accell siRNA appeared to diminish spontaneous inflammation in PCLuS as demonstrated by decreases in Illb (at $24 \mathrm{~h}$ ), Il6 (at $48 \mathrm{~h}$ ), and Tnfa (at $24 \mathrm{~h}$ ) mRNA expression. These effects were observed in PCLuS but not in PCKS. This could perhaps be caused by differences in the amount and type of immune cells present in both tissue types, as mentioned previously [6]. As these reductions are similar between non-targeting and Gapdh-targeting Accell siRNA, we hypothesize that Accell siRNA acts as an antagonist on siRNA sequence-independent proteins PKR and RIG-1, which are both present in the cytoplasm. It could be that the sterol modification on Accell siRNA inhibits the activation of PKR and RIG-1 through steric hindrance and, as a result, prevent activation of an immune signaling cascade. Nevertheless, the exact cause of immunosuppressive effects remains the subject of speculation. Further analysis was beyond the scope of this study.

\section{CONCLUSION}

In this study, we have demonstrated that PCLuS and PCKS can be successfully transfected with Accell siRNA to induce RNAi. Significant mRNA silencing was achieved without compromising the viability and structural integrity of the slices. Moreover, Accell siRNA did not induce an inflammatory response in either PCLuS or PCKS. As such, this ex vivo transfection model, which combines advantages of in vitro and in vivo models (e.g., simplicity, flexibility, throughput, and representability), could be used to decrease the expression of disease-related genes, to investigate the function of proteins, or to establish experimental models of disease in PCTS. Furthermore, PCTS can be prepared from human tissue which makes this transfection model particularly useful in translational research.

\section{ACKNOWLEDGEMENTS}

The authors thank Harry van Goor for the morphological evaluation of PCLuS and PCKS and Louise van Wijk for providing technical assistance during Western blotting analyses.

Open Access This article is distributed under the terms of the Creative Commons Attribution 4.0 International License (http://creativecommons.org/licenses/by/4.0/), which permits unrestricted use, distribution, and reproduction in any medium, provided you give appropriate credit to the original author(s) and the source, provide a link to the Creative Commons license, and indicate if changes were made.

\section{REFERENCES}

1. Hannon GJ. RNA interference. Nature. 2002;418:244-51. https://doi.org/10.1038/418244a.

2. Xia H, Mao Q, Paulson HL, Davidson BL. siRNA-mediated gene silencing in vitro and in vivo. Nat Biotechnol. 2002;20:1006-10. https://doi.org/10.1038/nbt739. 
3. Minakuchi Y, Takeshita F, Kosaka N, Sasaki H, Yamamoto Y, Kouno M, et al. Atelocollagen-mediated synthetic small interfering RNA delivery for effective gene silencing in vitro and in vivo. Nucleic Acids Res. 2004;32:e109. https://doi.org/10.1093/ nar/gnh093.

4. Urban-Klein B, Werth S, Abuharbeid S, Czubayko F, Aigner A. RNAi-mediated gene-targeting through systemic application of polyethylenimine (PEI)-complexed siRNA in vivo. Gene Ther. 2005;12:461-6. https://doi.org/10.1038/sj.gt.3302425.

5. de Graaf IAM, Olinga P, de Jager MH, Merema MT, de Kanter R, van de Kerkhof EG, et al. Preparation and incubation of precision-cut liver and intestinal slices for application in drug metabolism and toxicity studies. Nat Protoc. 2010;5:1540-51. https://doi.org/10.1038/nprot.2010.111.

6. Westra IM, Pham BT, Groothuis GMM, Olinga P. Evaluation of fibrosis in precision-cut tissue slices. Xenobiotica. 2013;43:98112. https://doi.org/10.3109/00498254.2012.723151.

7. Westra IM, Mutsaers HAM, Luangmonkong T, Hadi M, Oosterhuis D, de Jong KP, et al. Human precision-cut liver slices as a model to test antifibrotic drugs in the early onset of liver fibrosis. Toxicol. Vitr. 2016;35:77-85. https://doi.org/ 10.1016/j.tiv.2016.05.012.

8. Stribos EGD, Luangmonkong T, Leliveld AM, de Jong IJ, van Son WJ, Hillebrands J-L, et al. Precision-cut human kidney slices as a model to elucidate the process of renal fibrosis. Transl Res. 2016;170:8-16.e1. https://doi.org/10.1016/j.trsl.2015.11.007.

9. Starokozhko V, Vatakuti S, Schievink B, Merema MT, Asplund A, Synnergren J, et al. Maintenance of drug metabolism and transport functions in human precision-cut liver slices during prolonged incubation for 5 days. Arch Toxicol. 2017;91:2079-92. https://doi.org/10.1007/s00204-016-1865-x.

10. Ruigrok MJR, Frijlink HW, Hinrichs WLJ. Pulmonary administration of small interfering RNA: the route to go? J Control Release. 2016;235:14-23. https://doi.org/10.1016/ j.jconrel.2016.05.054.

11. Lynch I, Dawson KA. Protein-nanoparticle interactions. Nano Today. 2008;3:40-7. https://doi.org/10.1016/S17480132(08)70014-8.

12. Merz L, Höbel S, Kallendrusch S, Ewe A, Bechmann I, Franke $\mathrm{H}$, et al. Tumor tissue slice cultures as a platform for analyzing tissue-penetration and biological activities of nanoparticles. Eur J Pharm Biopharm. 2017;112:45-50. https://doi.org/10.1016/ j.ejpb.2016.11.013.

13. De Paula D, Bentley MVLB, Mahato RI. Hydrophobization and bioconjugation for enhanced siRNA delivery and targeting. RNA. 2007;13:431-56. https://doi.org/10.1261/rna.459807.

14. Hegde V, Hickerson RP, Nainamalai S, Campbell PA, Smith FJD, Mclean WHI, et al. In vivo gene silencing following noninvasive siRNA delivery into the skin using a novel topical formulation. J Control Release. 2014;196:355-62. https://oi.org/ 10.1016/j.jconrel.2014.10.022.

15. Mazzeo C, Gámez C, Rodriguez Marco A, de Zulueta A, Sanz V, Bilbao I, et al. Gene silencing of SOCS3 by siRNA intranasal delivery inhibits asthma phenotype in mice. PLoS One. 2014;9:e91996. https://doi.org/10.1371/journal.pone.0091996.
16. Whitehead KA, Dahlman JE, Langer RS, Anderson DG. Silencing or stimulation? siRNA delivery and the immune system. Annu Rev Chem Biomol Eng. 2011;2:77-96. https:// doi.org/10.1146/annurev-chembioeng-061010-114133.

17. Niehof M, Hildebrandt T, Danov O, Arndt K, Koschmann J, Dahlmann F, et al. RNA isolation from precision-cut lung slices (PCLS) from different species. BMC Res Notes. 2017;10:121. https://doi.org/10.1186/s13104-017-2447-6.

18. Merkel OM, Rubinstein I, Kissel T. siRNA delivery to the lung: what's new? Adv Drug Deliv Rev. 2014;75:112-28. https:// doi.org/10.1016/j.addr.2014.05.018.

19. van der Loos CM. Chromogens in multiple immunohistochemical staining used for visual assessment and spectral imaging: the colorful future. J Histotechnol. 2010;33:31-40. https://doi.org/ 10.1179/his.2010.33.1.31.

20. Bagasra O. Protocols for the in situ PCR-amplification and detection of mRNA and DNA sequences. Nat Protoc. 2007;2:2782-95. https://doi.org/10.1038/nprot.2007.395.

21. Halwer M, Nutting GC, Brice BA. Molecular weight of lactoglobulin, ovalbumin, lysozyme and serum albumin by light scattering 2. J Am Chem Soc. 1951;73:2786-90. https://doi.org/ 10.1021/ja01150a105.

22. Poosti F, Pham BT, Oosterhuis D, Poelstra K, van Goor H, Olinga $\mathrm{P}$, et al. Precision-cut kidney slices (PCKS) to study development of renal fibrosis and efficacy of drug targeting ex vivo. Dis Model Mech. 2015;8:1227-36. https://doi.org/ 10.1242/dmm.020172.

23. Dani C, Piechaczyk M, Audigier Y, Sabouty S, Cathala G, Marty L, et al. Characterization of the transcription products of glyceraldehyde 3-phosphate-dehydrogenase gene in HeLa cells. Eur J Biochem. 1984;145:299-304. https://doi.org/10.1111/j.14321033.1984.tb08552.x.

24. Franch HA. A mechanism regulating proteolysis of specific proteins during renal tubular cell growth. J Biol Chem. 2001;276:19126-31. https://doi.org/10.1074/jbc.M101777200.

25. Doherty MK, Hammond DE, Clague MJ, Gaskell SJ, Beynon RJ. Turnover of the human proteome: determination of protein intracellular stability by dynamic SILAC. J Proteome Res. 2009;8:104-12. https://doi.org/10.1021/pr800641v.

26. Sauer UG, Vogel S, Aumann A, Hess A, Kolle SN, Ma-Hock L, et al. Applicability of rat precision-cut lung slices in evaluating nanomaterial cytotoxicity, apoptosis, oxidative stress, and inflammation. Toxicol Appl Pharmacol. 2014;276:1-20. https:// doi.org/10.1016/j.taap.2013.12.017.

27. Behrsing HP, Furniss MJ, Davis M, Tomaszewski JE, Parchment RE. In vitro exposure of precision-cut lung slices to 2-(4-amino-3-methylphenyl)-5-fluorobenzothiazole lysylamide dihydrochloride (NSC 710305, Phortress) increases inflammatory cytokine content and tissue damage. Toxicol Sci. 2013;131:470-9. https://doi.org/10.1093/toxsci/ kfs319.

28. Iswandana R, Pham BT, van Haaften WT, Luangmonkong T, Oosterhuis D, Mutsaers HAM, et al. Organ- and species-specific biological activity of rosmarinic acid. Toxicol Vitr. 2016;32:2618. https://doi.org/10.1016/j.tiv.2016.01.009. 